\title{
THE ANTIPROTON DECELERATOR: AD
}

S. Baird, D. Berlin, J. Boillot, J. Bosser, M. Brouet, J. Buttkus, F. Caspers, V. Chohan, D. Dekkers,

T. Eriksson, R. Garoby, R. Giannini, O. Grobner, J. Gruber, J.Y. Hémery, H. Koziol, R. Maccaferri, S. Maury, C. Metzger, K. Metzmacher, D. Möhl, H. Mulder, M. Paoluzzi, F. Pedersen, J.P.

Riunaud, C. Serre, D.J. Simon, G. Tranquille, J. Tuyn, and A. Van der Schueren

CERN, CH-1211 Geneva 23, Switzerland

\section{Abstract}

A simplified scheme for the provision of antiprotons at $100 \mathrm{MeV} / \mathrm{c}$ based on fast extraction is described. The scheme uses the existing $\overline{\mathrm{p}}$ production target area and the modified Antiproton Collector Ring in their current location. The physics programme is largely based on capturing and storing antiprotons in Penning traps for the production and spectroscopy of antihydrogen. The machine modifications necessary to deliver batches of $1 \times$ $10^{7} \overline{\mathrm{p}} / \mathrm{min}$ at $100 \mathrm{MeV} / \mathrm{c}$ are described. Details of the machine layout and the experimental area in the existing AAC Hall are given.

\section{INTRODUCTION}

The scenario used up to the end of 1996 involved 4 machines downstream the antiproton production target: the Antiproton Collector (AC), the Antiproton Accumulator (AA), the Proton Synchrotron (PS) and the Low Energy Antiproton Ring (LEAR). They were used to collect, cool and decelerate antiprotons in the following sequence:

1) Antiprotons, produced by $26 \mathrm{GeV} / \mathrm{c}$ protons on the production target, are collected and precooled at $3.57 \mathrm{GeV} / \mathrm{c}$ in the AC.

2) They were transferred to the AA where they are accumulated and further cooled.

3) A bunch of a few $10^{9} \bar{p}$ was taken from the AA and sent to the PS every 30 minutes to several hours.

4) This bunch was decelerated in the PS from 3.57 to $0.6 \mathrm{GeV} / \mathrm{c}$.
5) It was then transferred to LEAR, where cooling (at 3 or 4 intermediate momenta) and deceleration brought the full intensity to low energy.

This scheme was designed as an 'annex' to the antiproton source for the $\operatorname{Sp} \overline{\mathrm{p}} \mathrm{S}$ (the SPS used as a $\overline{\mathrm{p}}-\mathrm{p}$ collider at $300 \mathrm{GeV}$ per beam). A simplified solution [1], using the modified AC and renamed AD (Antiproton Decelerator), is now being implemented. The antiproton physics programme will continue at the AD start in 1999, and LEAR will be converted into an ion accumulator for LHC [2].

\section{AD OVERVIEW}

The existing target area and the AC ring [3] in its present location (Fig.1) are used whereas the AA is dismantled. The basic AD cycle with the different intermediate levels is sketched in Fig. 2. After injection into the AD, bunch rotation is applied as in the AC to reduce the momentum dispersion of the antiproton batch. Then the beam is stochastically cooled at $3.5 \mathrm{GeV} / \mathrm{c}$ and $2 \mathrm{GeV} / \mathrm{c}$ and further decelerated in several steps. The next cooling level is at $300 \mathrm{MeV} / \mathrm{c}$ where the transverse emittances have grown to $33 \pi \mathrm{mm} \cdot \mathrm{mrad}$ and a $\Delta p / p$ of $0.2 \%$. Electron cooling is applied and, finally, after deceleration down to $100 \mathrm{MeV} / \mathrm{c}$, the beam is again electron-cooled. Beam characteristics and the cooling times are shown in Table 1.

The experimental area will be inside the AC ring. The shielding will be reinforced so that the users are allowed to access the area during $\bar{p}$ production and deceleration.

Table 1 - Transverse emittances and momentum spread before (i) and after (f) cooling, and cooling times. Only adiabatic increase due to deceleration is considered

\begin{tabular}{|c|c|c|c|c|c|c|}
\hline $\mathrm{p}$ & $\varepsilon_{i}$ & $\varepsilon_{f}$ & $\Delta p / p_{\text {i }}$ & $\Delta p / p_{f}$ & $t$ & Cooling process \\
\hline$[\mathrm{GeV} / \mathrm{c}]$ & \multicolumn{2}{|c|}{$[\pi \mathrm{mm} . \mathrm{mrad}]$} & \multicolumn{2}{|c|}{$[\%]$} & {$[\mathrm{s}]$} & \\
\hline 3.57 & 200 & 5 & 1.5 & 0.1 & 20 & \multirow{2}{*}{ Stochastic } \\
\hline 2.0 & 9 & 5 & 0.18 & 0.03 & 15 & \\
\hline 0.3 & 33 & 2 & 0.2 & 0.1 & 6 & \multirow{2}{*}{ Electron } \\
\cline { 1 - 5 } 0.1 & 6 & 1 & 0.3 & 0.01 & 1 & \\
\hline
\end{tabular}




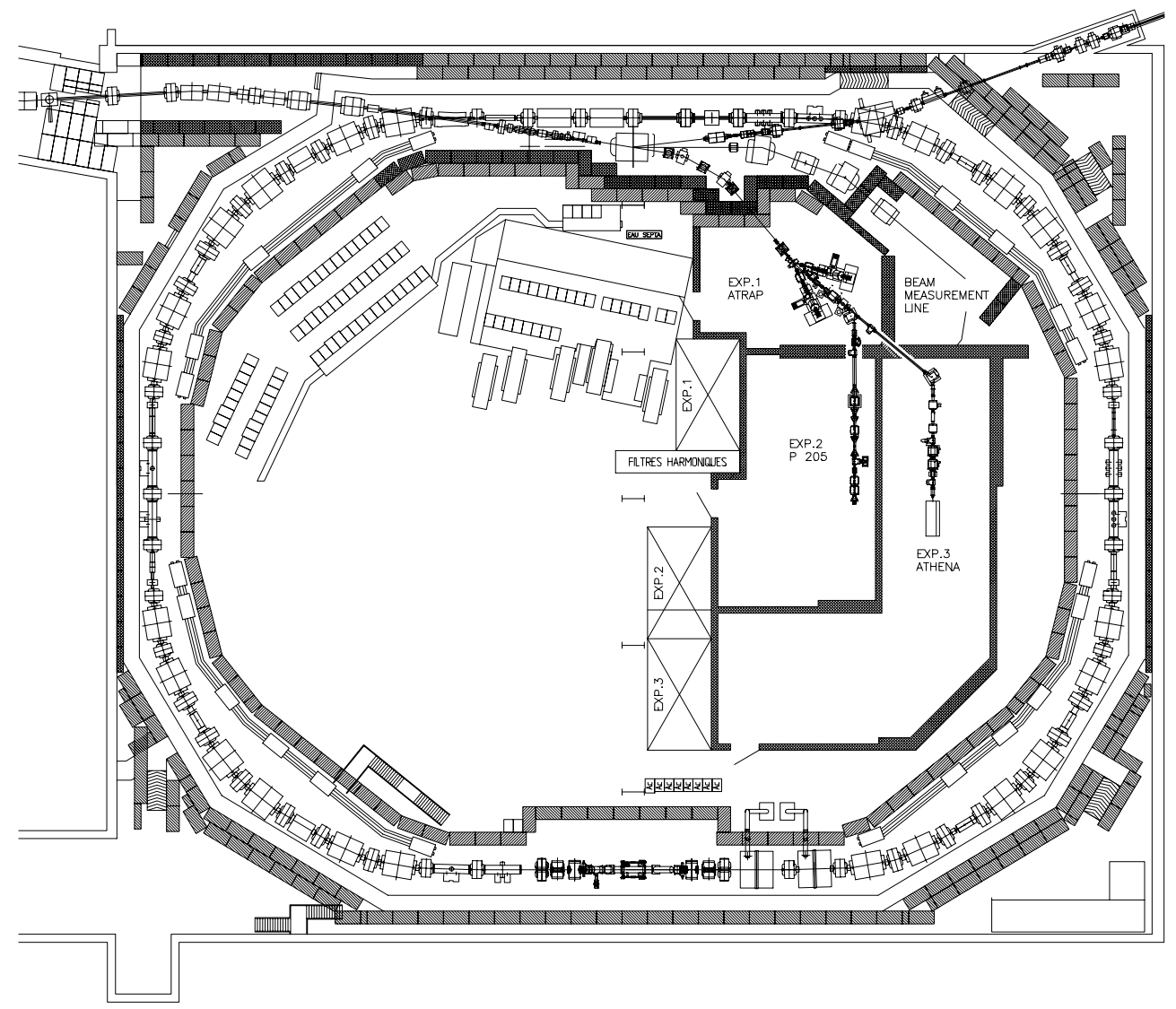

Fig. 1: AD layout.

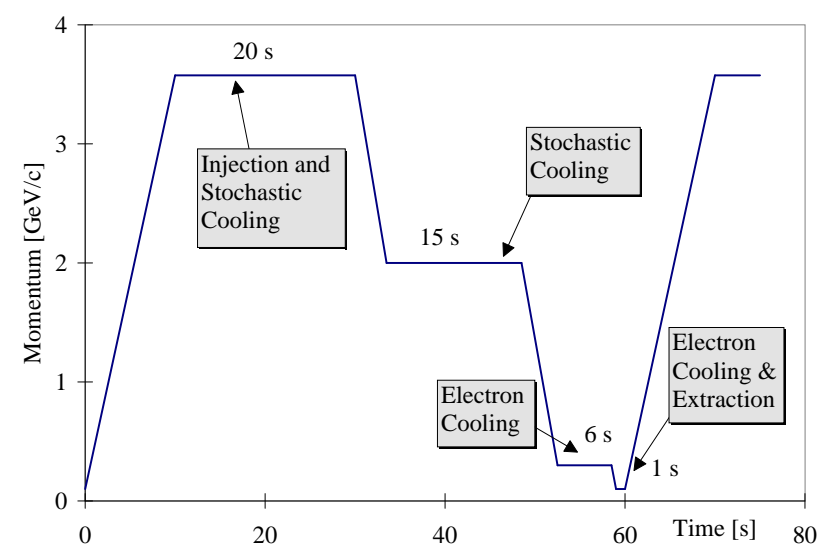

Fig. 2: Basic AD deceleration cycle.

Estimated characteristics of the ejected bunch are: $10^{7} \overline{\mathrm{p}}$ 's in one pulse of $0.2-0.5 \mu$ s length, with a repetition cycle of about 1 minute.

\section{AD LATTICE}

Detailed study [4] has led to the conclusion that suitable location for the electron cooling device is in a long straight section opposite to the injection section. The central quadrupole has to be removed. To reduce the strength of quadrupoles required for matching, it is proposed to add an additional quadrupole at the upstream and downstream end of the cooling insertion.

Phase advances between the pick-ups and kickers of the stochastic cooling have been adjusted by modifying the strength of other AD quadrupoles outside the cooling insertion. Further study to compensate the tune shift given by the solenoid of the electron cooler, calculations of the dynamical aperture are under way. The AD lattice functions is shown in Fig. 3. During study sessions made in the AC during 1996, it was found that orbit correction is necessary [4] to reach low momenta.

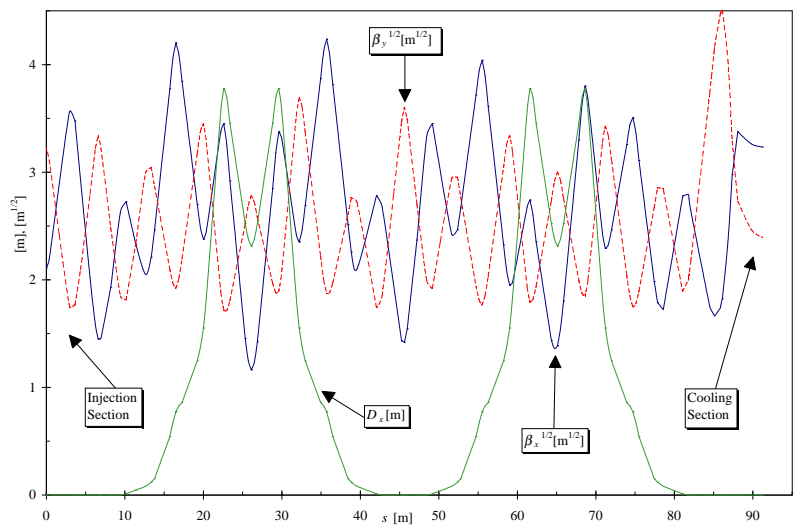

Fig. 3: The lattice functions of the $\mathrm{AD}$ where $\beta_{H}^{1 / 2}, \beta_{V}^{1 / 2}$ and $D$ are shown on half a ring starting in the middle of the injection section. 


\section{SYSTEMS INVOLVED}

\subsection{Antiproton Production}

The primary beam of $10^{13}$ protons at $26 \mathrm{GeV} / \mathrm{c}$ hitting the antiproton production target will be similar to the present one. It will, however, benefit from developments carried out in view of the use of the PS as part of the LHC injector chain [5].

Antiprotons emerging at $3.5 \mathrm{GeV} / \mathrm{c}$ from the target will be focused and matched to the transport line acceptance of $200 \pi \mathrm{mm} \cdot \mathrm{mrad}$ by a magnetic horn pulsed at $400 \mathrm{kA}$.

\subsection{Radiofrequency Systems}

The $5 \times 10^{7} \overline{\mathrm{p}}$ 's injected in the AD will be trapped by the existing 9.5 MHz $(h=6)$ rf system, as in the present scheme, and a bunch rotation will be applied in order to reduce the energy spread. The beam will then be decelerated with the present $1.6 \mathrm{MHz}(h=1)$ rf system whose frequency range will be extended down to $0.5 \mathrm{MHz}$ and changes of harmonic numbers at intermediate energy levels will be performed.

This rf system will also be used to shorten the bunch at $100 \mathrm{MeV} / \mathrm{c}$ prior to extraction

\subsection{Beam Cooling Systems}

Stochastic cooling is needed at $3.57 \mathrm{GeV} / \mathrm{c}$ and $2 \mathrm{GeV} / \mathrm{c}$ (Fig. 1), for which band I (0.9 to $1.6 \mathrm{GHz})$ and band II (1.6 to $2.4 \mathrm{GHz}$ ) of the present systems will be employed. The pick-ups and kickers of band I remain in their present location. The band II system will be located in the present band III location. At $2 \mathrm{GeV} / \mathrm{c}$ band I is used to compensate the adiabatic beam blow-up due to the deceleration. The pick-up sensitivity is reduced by a factor of 2 compared to $3.57 \mathrm{GeV} / \mathrm{c}$. Band III (2.4 to $3.2 \mathrm{GHz}$ ) is not used as the gain in the cycle time would not be significant and space is needed for the electron cooling system.

Electron cooling will be applied at low momenta, especially at 300 and $100 \mathrm{MeV} / \mathrm{c}$ (Fig. 2). With only minor modifications, the existing LEAR cooler can be used in the AD.

\subsection{Vacuum}

Amongst the different effects of the residual gas which have an influence on the quality of the antiproton beam, both the single scattering loss and the multiple scattering blow-up become very important at low momenta.

In the presence of cooling, with a time constant of $1 \mathrm{~s}$ for the large beam, an equilibrium emittance of $\sim 20 \pi \mathrm{mm} \cdot \mathrm{mrad}$ would be reached with the vacuum conditions of AC. For efficient capture of antiprotons in a
Penning trap, equilibrium emittances $<1 \pi \mathrm{mm} \cdot \mathrm{mrad}$ at $100 \mathrm{MeV} / \mathrm{c}$ are important. Therefore, an improvement of the present vacuum conditions by about a factor 20 is required.

\subsection{Power Converters}

In order to guarantee a current stability at low energy, active filters must be added on the main power converters. The trimming power supplies used in the AC will have to run below the present minimum controllable current. It is proposed to build new power converters which will be stable down to a very small current.

\section{OPERATION}

During setting-up with protons, the radiation level induced by a beam of $3 \times 10^{10}$ protons will be too high to allow access to the hall. Therefore, the hall and the AD ring will be considered as primary zone. During normal $\overline{\mathrm{p}}$ operation, the new shielding configuration will reduce the dose rate in the hall; in this case it will be considered as a secondary zone.

\section{CONCLUSION}

The modified $\mathrm{AC}$ as an antiproton decelerator will deliver a dense beam of $10^{7} \overline{\mathrm{p}}$ every minute at $100 \mathrm{MeV} / \mathrm{c}$ with bunch lengths between 200 and 500 ns. This simplified scheme opens the possibility for an antiproton physics programme based on fast extracted beams. CERN will provide the framework for the $\mathrm{AD}$ but cost and manpower for the project will be provided by the user laboratories, who also will help with the operation.

\section{REFERENCES}

[1] AD Study Group, S. Maury (editor) : 'Design Study of the Antiproton Decelerator: AD', CERN/PS 95-43 (AR), 1995.

[2] The LHC Study Group: 'The Large Hadron Collider: Conceptual Design', CERN/AC 95-05 (LHC), 1995.

[3] ACOL Study Group, E. Wilson (editor): 'Design Study of an Antiproton Collector for the Antiproton Accumulator (ACOL)', CERN 83-10, 1983.

Koziol and S. Maury: 'Parameter List for the Antiproton Accumulator Complex (AAC)', Edition 1994, CERN/PS 95-15, 1995.

[4] P. Belotschskii, C. Carli, T. Eriksson, R. Giannini, S. Maury, D. Möhl, and F. Pedersen: 'Beam Optics Issues for the Antiproton Decelerator (AD)', this conference.

[5] R. Cappi, R. Garoby, S. Hancock, M. Martini, J.P. Riunaud, K. Schindl, H. Schonauer, 'Beams in the PS Complex During the LHC Era', CERN/PS 93-08 (DI) Rev., 1993. 